In: 1999 IEEE International Symposium on Assembly and Task Plan-

ning (ISATP'99), Porto, Portugal, July 21-24, 1999.

\title{
Manipulating deformable linear objects - Contact states and point contacts -
}

\author{
*Institute for Process Control and Robotics \\ (IPR), University of Karlsruhe, D-76128 \\ Karlsruhe, Germany \\ e-mail:dHenrich@ira.uka.de \\ http: //wwwipr.ira.uka.de/ dhenrich/
}

Dominik HENRICH* ${ }^{*}$ Tsukasa OGASAWARA*, and Heinz WÖRN*

\begin{abstract}
The task of handling non-rigid one-dimensional objects by a robot manipulation system is investigated. To distinguish between different non-rigid object behaviors, five classes of deformable objects from a robotic point of view are proposed. Additionally, an enumeration of all possible contact states of one-dimensional objects with polyhedral obstacles is provided. Finally, the qualitative motion behavior of linear objects is analyzed for stable point contacts. Experiments with different materials validate the analytical results.
\end{abstract}

\section{Introduction}

The manipulation of rigid objects by robots has been investigated for several decades. Less work has been done investigating the manipulation of nonrigid or deformable objects. Here, we focus on the one-dimensional or linear deformable objects, such as cables, wires, ropes, strings, beams, etc. This task has various application fields, for example, hot-wire maintenance [Nakashima95], cable form assembly, or production of control cabinets. The main problem of manipulating these objects is that they may change their shape during manipulation.

To cope with this problem, one approach is to estimate the shape of the deformable objects by calculating an internal model and simulate the object behavior. A static model for objects and obstacles can be calculated in two [Hirai94] or three [Wakamatsu95] dimensions. An extension leads to a dynamic model of deformable linear objects [Wakamatsu97]. On the one hand, the object shape can be calculated with these methods precisely (direct simulation problem). On the other hand, it is not clear how to use the object models to control the robot motion, that is, to solve the inverse simulation problem. Additionally, the shape calculation can be very time consuming.

Another approach is to employ sensor systems to detect the object's shape. Vision systems can be used for example to guide the robot motion while making a knot into a rope [Inoue83] or to detect the shape of a flexible beam while inserting into a hole [Zheng91, Nakagaki96]. Force/torque sensors can

\author{
"Nara Institute of Science and Tech- \\ nology (NAIST), Graduate School of \\ Information Science, Nara, Japan, \\ e-mail: ogasawar@ is.aist-nara.ac.jp \\ http: //cairo.aist-nara.ac.jp/
}

be used to detect buckling while inserting flexible beams into a hole [Nakagaki95, Kraus97]. Same as with the model-based approaches, the sensor-based approaches are quantitative ways to measure or calculate the shape.

A qualitative sensor-based approach to manipulate deformable linear objects is skill-based manipulation. Manipulation skills are motion primitives to achieve a particular target state of the manipulated object. They are specified in the task domain independently of the robot hardware and hide control procedures and sensor feedback to the programmer. Skills are robust and overcome residual errors and uncertainties in both, models and manipulator movements. For example, for rigid polyhedral objects, the manipulation skills serve as transitions between contact states and simplify programming of a model-based manipulation system [Hasegawa92]. The sequence of manipulation skills can then be extracted automatically from the motion performed by an operator in a simulator [Onda95].

The basic precondition of skill-based manipulation is the identification of object states. Then, the manipulation skills may serve as transitions between these states. The question is what kind of state models can be used for deformable objects. Topological states, such as provided by the knot theory, use the number and kind of crossings of the linear object (with itself) [Crowell77]. For rigid objects, contact states differ in the involved type and number of geometric primitives [Suehiro89]. Shape states are determined by calculating the precise or approximate object geometry [Wakamatsu95, Higashijima98] and can hardly be distinguished in a symbolic way. Position states use the location and orientation of geometric primitives relative to other geometric primitives [Morrow97].

In this paper, we investigate contact states and point contacts of deformable linear objects. The results may serve as the first steps to apply the concept of skill-based manipulation to deformable linear objects. For this, we have to come up with an answer to the following questions: How can deformable objects be classified from a robotic point of view (Section 2)? What are the possible 
contact states of deformable linear objects (Section 3)? How do deformable linear objects in contact move qualitatively (Section 4)? What are the conclusions and future work (Section 5)?

\section{Object classification}

When handling non-rigid objects, one has to face the problem that there are many different kinds of properties of object material. They result in different approaches to handle the objects by robots. Thus, it is helpful to distinguish between the behavior of different deformable objects. Here, we propose five classes of deformable objects from a robotic point of view. Especially, the qualitative issues are regarded.

There have been done various works in this direction in material science [Fung65]. Our classification is based on this work. For robotic application, additional properties have to be considered. This is due to the fact that in robotic applications the objects are moved. Especially, a change in the object orientation is important.

For the many materials occurring in robot handling applications, there are two basic types of deformations: The elastic deformation disappears after forces relax. The plastic deformation remains after forces relax. The viscous deformation is not regarded here. Five deformation classes based on these definitions are introduced in the following.

When handling a deformable object, it is important to have some indication in which way its shape will change. For classifying different objects or their material, we use two important measures, the applied force and the resulting deformation, which are regarded only qualitatively.

For the force applied to the object, it is sufficient to regard only the amount $|F|$ of force because we assume an isotrop material, thus, the direction of the force can be neglected. Additionally, $|F|$ ranges within some minimum force and maximum value $F_{\min }$ and $F_{\max }$, respectively, because of the robot's mechanical constraints. Within this range, the gravitation force is important. All these values are constant for one robot but may differ for different robots.

\begin{tabular}{|c|c|c|c|c|c|}
\hline & $\mathbf{N}$ & E- & $E+$ & $\mathbf{P}-$ & $\mathbf{P +}$ \\
\hline Description & no deformation & $\begin{array}{l}\text { low elastic } \\
\text { deformation }\end{array}$ & $\begin{array}{l}\text { high elastic } \\
\text { deformation }\end{array}$ & $\begin{array}{l}\text { low plastic } \\
\text { deformation }\end{array}$ & $\begin{array}{l}\text { high plastic de- } \\
\text { formation }\end{array}$ \\
\hline $\begin{array}{l}\text { Linear metal ob- } \\
\text { jects }\end{array}$ & $\begin{array}{l}\text { short steal } \\
\text { tubes }\end{array}$ & $\begin{array}{l}\text { short spring } \\
\text { steal }\end{array}$ & $\begin{array}{l}\text { long spring } \\
\text { steal }\end{array}$ & $\begin{array}{l}\text { short iron } \\
\text { wires }\end{array}$ & ropes \\
\hline $\begin{array}{l}\text { Geometry de- } \\
\text { pends on }\end{array}$ & nothing & $\begin{array}{l}\text { current con- } \\
\text { tacts }\end{array}$ & $\begin{array}{l}\text { current con- } \\
\text { tacts + orien- } \\
\text { tation }\end{array}$ & $\begin{array}{l}\text { current con- } \\
\text { tacts + former } \\
\text { contacts }\end{array}$ & $\begin{array}{l}\text { current contacts } \\
\text { + orientation, } \\
\text { former contacts }\end{array}$ \\
\hline Handling task & simple & & $\ldots$ & & complex \\
\hline
\end{tabular}

Figure 2: Deformation classes with example objects and geometric properties (Deformation classes sorted by increasing complexity for handling tasks)
For the resulting deformation of the object, we restrict ourselves to some scalar measure $|D|$ of the deformation, thus, the object shape is neglected. Additionally, $|D|$ ranges within some $D_{\min }$ and $D_{\max }$. Below $D_{\text {min }}$ the deformation is not important for the considered application and beyond $D_{\max }$ the object may be destroyed. Within this range, the deformation amount $D_{\text {rev }}$ indicates the limit, where the reversible (elastic) deformation changes to an irreversible (plastic) one. All these values are constant for a specific material and for a specific application but may change for different applications.

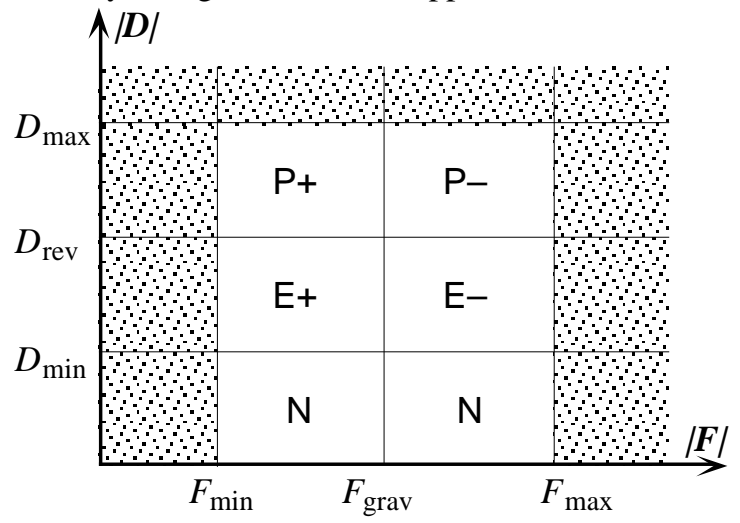

Figure 1: The different deformation classes $(\mathrm{N}, \mathrm{E}-$, $\left.\mathrm{E}_{+}, \mathrm{P}_{-}, \mathrm{P}_{+}\right)$depending on the applied force amount $|F|$ and on the resulting amount of deformation $|D|$

Based on these two measures, the following five deformation classes can be distinguished (see Figure 1). Objects with deformations less than $D_{\text {min }}$ (e.g., short steal tubes) are called non-deformable and belong to the class N. Between $D_{\text {min }}$ and $D_{\text {rev }}$, elastic deformation takes place. If a force less than gravity already results in an elastic object deformation, then this object is called highly elastic deformable and belongs to class E+. Thus, objects in $\mathrm{E}+$ (e.g., long spring steal) may change their shape if the robot gripper grasping the objects changes its orientation. If a force greater than gravitation is necessary for an elastic object deformation, then the objects will not change their shape due to a change in orientation (e.g., short spring steal). Thus, these objects are called low elastic deformable and belong to class E-. 


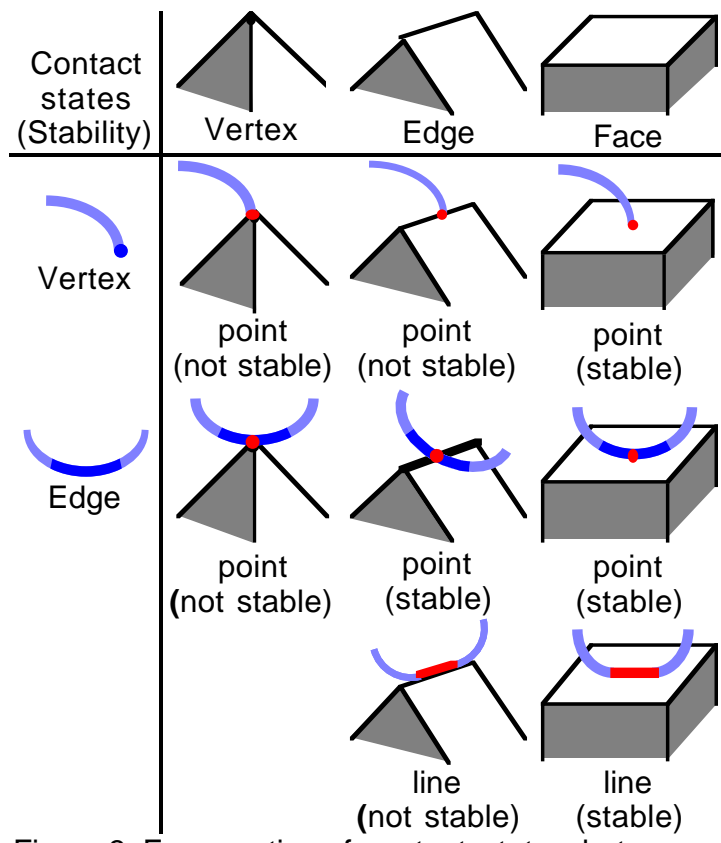

Figure 3: Enumeration of contact states between a deformable linear object and a convex polyhedron based on the geometric primitives

Between $D_{\text {rev }}$ and $D_{\text {max }}$, plastic deformation takes place and the classification is analog to the case of elastic deformation. Thus, highly and low plastic deformable objects belong to classes $\mathrm{P}+$ and $\mathrm{P}-$ and will deform if a force smaller and greater than gravity is applied (e.g., ropes and short iron wires), respectively.

A summary of the different deformation classes and corresponding example objects is given in Figure 2. Please note that some materials may belong to multiple classes depending on the applied force. The shape of an object can depend only on the obstacle contacts or additionally on the orientation of the object. This is the intuitive difference between classes $\mathrm{X}+$ and $\mathrm{X}-, \mathrm{X} \in\{\mathrm{E}, \mathrm{P}\}$. Furthermore, the shape can depend only on the current state or additionally on the former states. This is one difference between classes $\mathrm{E} \pm$ and $\mathrm{P} \pm$. In Figure 2, these influence factors on the object geometry are listed for each deformation class. The more shape influencing factors exist, the more complex is the handling task. Thus, the number of factors indicates the complexity of the deformation classes. This results in $\mathrm{N}$ containing the easiest objects and $\mathrm{P}+$ the most difficult objects concerning their automatic handling by robots.

\section{Contact states}

A very frequent task of robot manipulators is to achieve a contact situation of one object with some other object(s). To solve this type of tasks for deformable objects, it is important to know what

\begin{tabular}{|c|c|c|}
\hline Description & Illustration & $\begin{array}{c}\text { Primitives } \\
\text { (contact type) }\end{array}$ \\
\hline $\begin{array}{l}\text { lying on } \\
\text { table }\end{array}$ & & $\begin{array}{l}\text { edge/face } \\
\text { (line) }\end{array}$ \\
\hline hooked & & $\begin{array}{l}\text { edge/edge } \\
\text { (point) }\end{array}$ \\
\hline curled & & $\begin{array}{l}1 \text { edge/edge } \\
\text { (point) }\end{array}$ \\
\hline knot & & $\begin{array}{l}3 \text { edge/edge } \\
\text { ( } 3 \text { points) }\end{array}$ \\
\hline $\begin{array}{l}\text { beam-in- } \\
\text { tube }\end{array}$ & & $\begin{array}{l}3 \text { edge/edge } \\
\text { ( } 3 \text { lines) }\end{array}$ \\
\hline $\begin{array}{l}\text { beam-in- } \\
\text { hole }\end{array}$ & & $\begin{array}{l}3 \text { edge/edge } \\
1 \text { vertex/face } \\
\text { ( } 3 \text { lines and } 1 \\
\text { point) }\end{array}$ \\
\hline
\end{tabular}

Figure 4: 3D-Examples for single and multiple stable contact states with the involved geometric primitives and resulting contact types

kinds of contact situations are possible and how to achieve them. Therefore, we provide in this section an enumeration of all possible contact states and give some examples.

According to the increasing complexity of the deformation classes in the previous section, we will start with the simplest deformable linear objects, that is, objects in class E-. Additionally, we restrict ourselves to investigate deformable linear objects in contact only with polyhedral and rigid objects (called "obstacles" in the sequel). Finally, we focus on geometry and kinematics, thus, neither friction nor forces are regarded. This leads us to a more qualitative point of view.

The situation of two objects in contact is mainly characterized by the object shapes. The object shape itself can be characterized by a set of geometric primitives. For polyhedral objects, these primitives are vertices, edges and faces. For linear objects, we regard the tips at both ends each as a vertex and the peace in-between as one edge. This model holds true for most thin linear objects. In the case that the cross-section cannot be neglected, the object is not linear anymore and has to be modeled, for example, as a (general) cylinder.

When primitives of a polyhedral obstacle and a linear object are in contact, different contact states can be observed. An enumeration of all possible contact states is given in Figure 3. Within these contact states, two different contact types can be distinguished: point contact and line contact. They are characterized by the dimension ( 0 or 1$)$ of the contact area of the two touching primitives. 


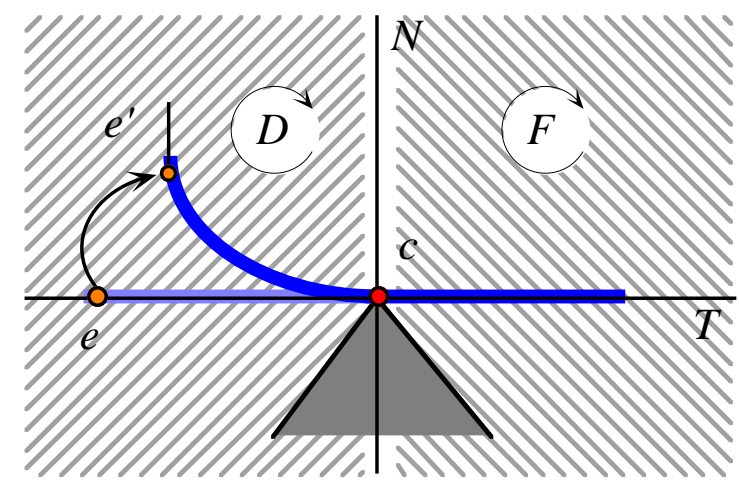

Figure 5: A deformable linear object having point contact with an obstacle in $c$ with tangent $T$ and normal $N$, and the deformation from $e$ to $e^{\prime}$ without motion in $C$

The contact states have different properties concerning their stability. For better identification of these properties, we may think of a linear object being deformed to some extend, while having contact with an obstacle. Then, a contact state is sta$b l e$, if a small movement of the object in any direction will not change the contact state. Otherwise, a contact state is not stable. For handling deformable objects, the stable contact states are important.

After introducing the contact states and their properties, the question is whether they are defined in a useful way. We can give a positive answer by providing various examples for contact situations in Figure 4. The examples show that the introduced contact states can be easily used to characterize very different contact situations with single or multiple contacts. These situations include contacts of the linear object with other (rigid) obstacles as well as contacts with itself. Additionally, compared to the other state models mentioned in Section 1, the contact states capture all important aspects for handling deformable linear objects by robot manipulators.

Concerning the contact states in general, the objects can perform different types of motion. For a rigid object, there are the contact and free motion establishing and releasing the contact state, respectively. The rotate or slide motion does not change the contact state but changes the orientation or position of the object, respectively. For deformable objects, additionally, the deform motion changes the shape of the object.

\section{Point contacts}

To enable a robot manipulator to handle deformable linear objects automatically, it is important to know how the object behaves when being in contact with some obstacles. Here, we are interested mainly in the qualitative behavior. Thus, in the

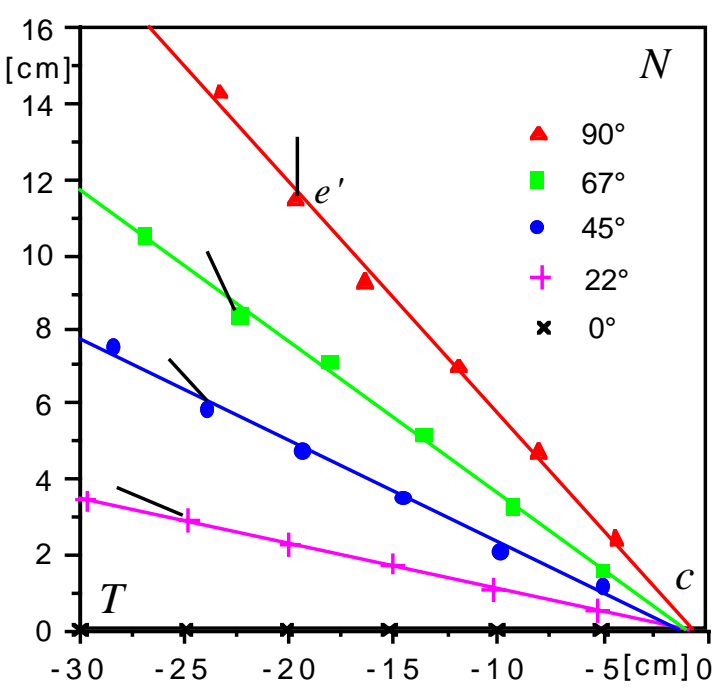

Figure 6: Position $e^{\prime}$ of effect point $e$ after rotating the linear object in $e$ and adjusting the location of $e$ such that there is no motion in the contact point $c$ (orientation in $e^{\prime}$ indicated by small line)

following sections, we will answer questions like: What is the resulting motion of one end of a linear object if the other end is moved? For rigid objects, the answer is rather simple but not so for deformable objects.

The qualitative motion behavior of linear objects is investigated here for stable point contacts. Unstable contact states are only intermediate and finally lead to stable ones. Line contacts have a restricted motion behavior compared to point contacts. Especially, we concentrate on edge/edge point contacts, which give a good illustration of the effects. Analogous investigations can be performed for other stable contact states. All investigations are illustrated by a projection of the objects onto a 2-dimensional plane orthogonal to the obstacle edge.

\subsection{Motion type areas}

An object can be moved in space by translations, rotations, or any combination of those. Rotations can be characterized by the rotation center and rotation angle. Translations can be viewed as a special case of (generalized) rotations with the rotation centers lying in infinity perpendicular to the direction of the translation. It is sufficient to regard only clock-wise rotations since the results for counter-clockwise rotations can be retrieved by a mirrored setup along a vertical symmetry axis.

The basic setup of a deformable linear object having point contact with a rigid polyhedral obstacle in point $c$ is shown in Figure 5. The axis $T$ indicates the tangent of the linear object in $c$. The axis $N$ indicates the normal to $T$ in $c$. These axes form a local coordinate system $T \times N$. 


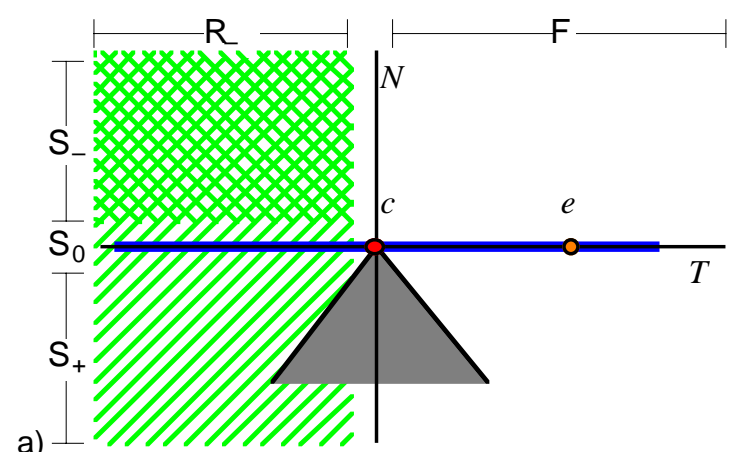

Figure 7: The resulting motions in contact point $c$ for different rotation center areas for effect point $e$ located to the right of $c(a)$ and to the left of $c(b)$

When moving the deformable linear object by a generalized rotation, different resulting motion types can be observed. They depend on the location $x$ of the rotation center in $T \times N$. Since moving the linear object may rotate $T \times N$, the resulting motion types hold true only for infinitesimal small motions or "instantaneous" rotation centers. The rotation centers can be clustered to the following areas:

$x \in\{c\}:$ keeps contact with rotation in $c$ (rotate) $x \in M c$ : keeps contact with translation in $c$ (slide) $x \in F W$ : detach for clock-wise rotation (free) $x \in D W$ : deform for clock-wise rotation (deform)

For the rotation center areas of the rotate, slide, and free/contact motions, deformable objects behave in the same way as rigid objects. Since rigid objects and their motion types have already been investigated earlier in [Suehiro89], it is sufficient to concentrate on the deform motion added for deformable objects. This will be done in the next sections for straight linear objects.

\subsection{Deformation without motion}

A deformation motion leads to a change in shape without changing the contact state. Thus, it does not necessarily lead to a movement in the contact point $c$. This effect occurs only for deformable objects and, therefore, will be investigated in this section.

Our experimental setup for deformations without motion of a linear object with contact point $c$ is illustrated in Figure 5. Contrasting with motions of rigid objects, here, the motion types depends additionally on the location of the effect point $e$ of the motion. The position and orientation of the effect point before and after moving the object is indicated by $e$ and $e$, respectively.

The experimental results for an electric cable belonging to class E- (Yazaki, 1997, $0.5 \mathrm{~mm}^{2}, 4$ wires) are shown in Figure 6. They are based on the setup in Figure 5, indicated by $c, T$, and $N$. The linear object and the obstacle are hidden for clarity. In each effect point $e$ with a certain distance to the contact point $c$, we fix the orientation of the linear object and adjust the location of the resulting $e^{\prime}$ in

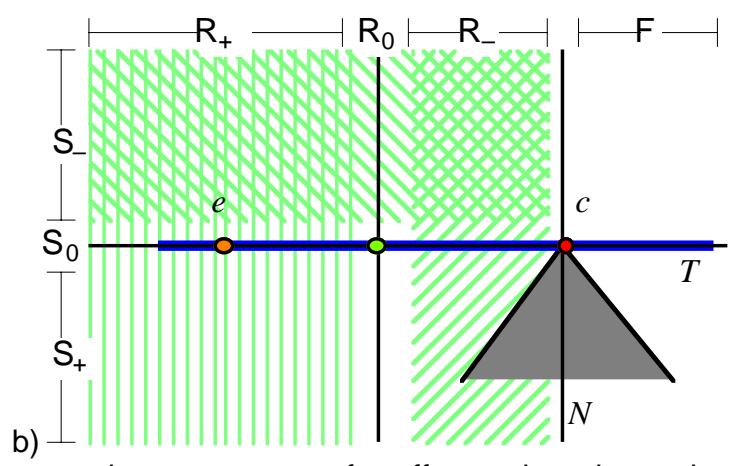

the $2 \mathrm{D}$ plane such that the linear object does neither rotate nor slide in $c$.

The resulting effect points $e^{\prime}$ with corresponding orientation are located on a line pointing to the contact point $c$. This effect was validated by our simulation system for deformable linear objects based on [Wakamatsu97]. Additionally, we have determined the corresponding rotation centers by geometric construction. The resulting rotation centers for each distance between $e$ and $c$ are located in clusters along $T$.

There are two applications of these results: First, a robot manipulator may deform the linear object without any movement in $c$ by rotating around the corresponding rotation center. Second, if friction can be neglected, a robot manipulator may slide a linear object without changing the orientation in the contact point $c$ by moving along a straight line through $c$ in Figure 6.

\subsection{Deformation with motion}

Similar to rigid objects, deformable objects may have a resulting motion in the contact point if they are moved. Additionally, deformable objects may change their shape during this motion. This can lead to some surprising effects for the resulting motion in the contact point, which are investigated in this section.

As before, we are interested only in the qualitative motion behavior of the linear object in the contact point. In this point, it is reasonable to distinguished the basic motion types slide and rotation a bit further. The slide motion is subdivided into left slide $\left(S_{-}\right)$, right slide $\left(S_{+}\right)$and no slide $\left(\mathrm{S}_{0}\right)$. The rotation motion is subdivided into negative or clock-wise rotation $\left(\mathrm{R}_{-}\right)$, positive or counter-clockwise rotation $\left(\mathrm{R}_{+}\right)$, and no rotation $\left(R_{0}\right)$. The three slide and rotation motions can be determined independently.

Since for deformable objects, the location of the effect point $e$ is important, we have to distinguish between $e$ lying to the left or to the right of the contact point $c$. Please remember that it is sufficient to regard only the area with the deform mo- 

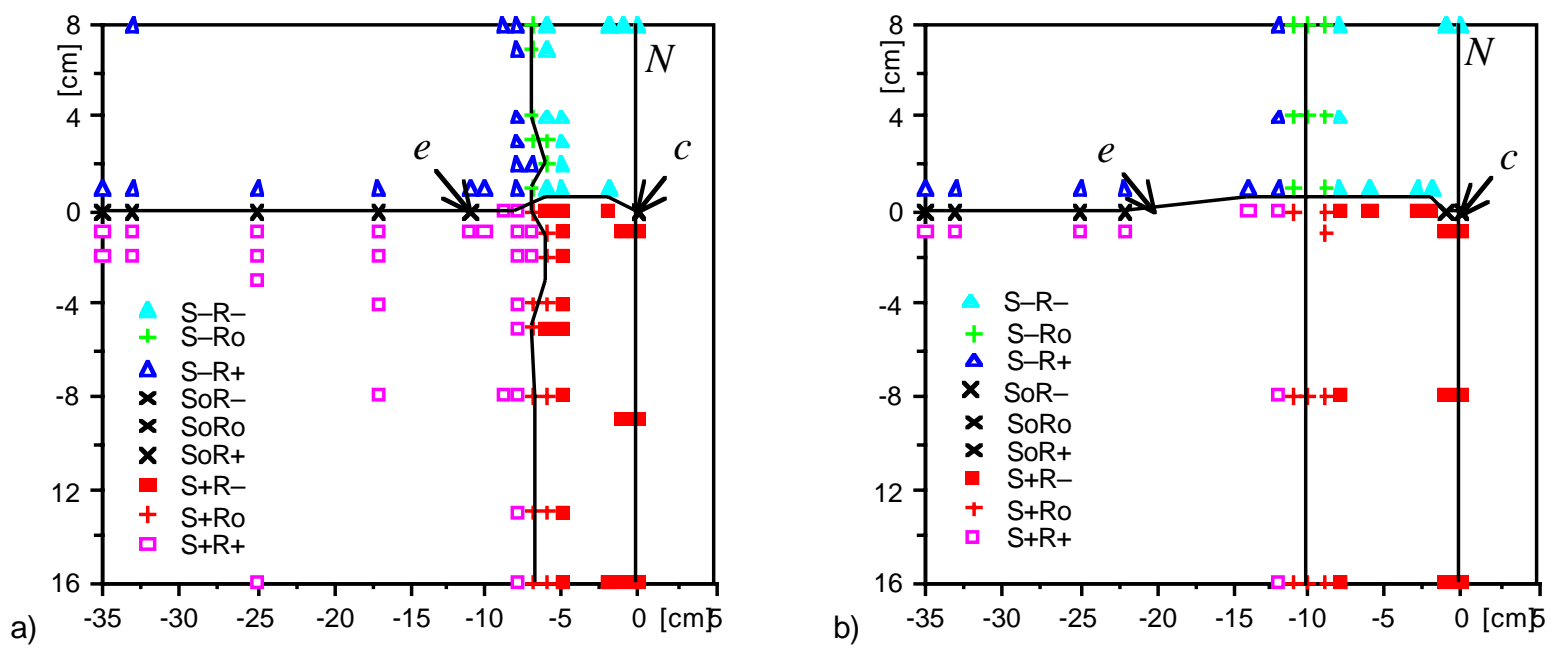

Figure 8: Different rotation centers and the resulting motion in contact point $c$ for a clockwise rotation of effect point $e$ lying to the left of $c$ in $10 \mathrm{~cm}$ distance (a) and $20 \mathrm{~cm}$ distance (b)

tion type (rotation center left to $N$ ), which is specific for deformable objects.

Let us assume that $e$ is right of $c$ as illustrated in Figure 7a. As soon as $e$ is moved downwards, the linear object will rotate clockwise in $c$, which holds true for rotation centers left of $N$ (where the main motion component is a downwards translation). If $e$ is additionally moved to the left or to the right, this results in a left or right slide, which holds true for rotation centers above or below $T$, respectively. A rotation center lying on $T$ results in no slide $\left(S_{0}\right)$, because there is no left or right movement of $e$. Please note that, for a small movement with the rotation center on $T$, the linear object at first only rotates in $c$. At the same time, the tangent rotates too, and the (unchanged) rotation center is moved below the tangent. Then, the linear objects slides additionally to the right in $c$. Thus, $\mathrm{S}_{0}$ is a small rotation center area forming a transition between $S_{-}$and $S_{+}$.

Now, let us assume that $e$ is left of $c$ as illustrated in Figure $7 b$. The rotation center areas for the left, right, and no slide are the same as for $e$ being to the right of $c$. On the one hand, if $e$ is additionally moved downwards the linear object will rotate counter-clockwise in $c\left(\mathrm{R}_{+}\right)$, which (at least) holds true for rotation centers left of $e$. On the other hand, if the rotation center lies on ${ }^{1} N$ and especially in $c$, the linear object will rotate clockwise in $c$ ( $\left.\mathrm{R}_{-}\right)$. Thus, similar to the slide transition, there has to be a transition area $\mathrm{R}_{0}$ between $\mathrm{R}_{-}$and $\mathrm{R}_{+}$(between $c$ and $e$, respectively). Since the setup and the slide behavior are symmetrical to the horizontal axis $T$, there is a strong indication that the transition area $\mathrm{R}_{0}$ is a line orthogonal to $T$.

1 Here, rotation centers in infinity are excluded, which lead only to slide motions.
This rather surprising effect is validated by some experimental results in Figure 8. As deformable linear object, we used a pneumatic hose (Polyurethane, $4 \mathrm{~mm} / 6 \mathrm{~mm}$ inner/outer diameter). For the same setup as in Figure $7 b$, we chose different rotation centers, rotated the effect point $e$ clockwise, and observed the resulting motion of the linear object in the contact point $c$. In the figure, the resulting rotation center areas are separated by lines. Besides of some small measuring artifacts, the motion behavior is the same as predicted in Figure 7b. This experiment is conducted for a distance between $c$ and $e$ of $10 \mathrm{~cm}$ and $20 \mathrm{~cm}$ in Figure $8 \mathrm{a}$ and $\mathrm{b}$, respectively. The vertical transition line between $\mathrm{R}_{-}$and $\mathrm{R}_{+}$seems to be at a certain proportion between $c$ and $e$.

We have performed further experiments, which are not shown here. One kind assumes the more simple case of Figure 7a. Another kind uses other linear objects of deformation class E-, such as spring steal (0.5 mm x $18 \mathrm{~mm}$ cross-section) or electrical cable (Yazaki, 1997, $0.5 \mathrm{~mm}^{2}, 4$ wires). A last kind investigates objects in $\mathrm{P}-$ (carbon wire, $2 \mathrm{~mm}$ diameter). Additionally, we executed all motions types of Figure $7 \mathrm{~b}$ by an industrial robot manipulating a pneumatic hose. All experimental results validate the presented analysis.

\section{Conclusions and future work}

As conclusion, we have experienced during our work that the introduced classification of deformation classes helps very much to distinguish different object materials and the behavior to be expected. Additionally, the presented specification of contact states of linear objects with polyhedral obstacles provides a good basis to develop manipulation skills for deformable objects. Finally, the 
analysis of most frequent (point) contact state shows that a qualitative motion behavior of linear objects can be recognized, which may be exploited for manipulation skills. Thus, this seems to be a promising qualitative approach for robust manipulation of deformable linear objects avoiding the explicit modeling and calculation of the object shape.

The results for single point contacts can easily be used for multiple point contacts. For this, rotation center areas of the single contacts can be overlapped according to the actual situation. This holds true even if the effect points lie on different sides. To achieve an overall motion of the linear object, the rotation center has to be chosen from the overlapping area of the single contact points yielding the necessary motion in each contact.

Currently, we are developing the corresponding manipulation skills for deformable linear objects. Future work will include the investigation of the remaining contact states, such as edge contacts.

\section{Acknowledgments}

We would like to thank Dr. H. Tsukune and the Robotics Research Group at the Electrotechnical Laboratory, AIST, MITI, where the main part of this work was performed. The work was supported in the framework of a STA fellowship by Dominik Henrich granted from the Science and Technology Agency, Japan. Many thanks to Axel Remde at the IPR for the valuable discussions and proofreading.

\section{References}

[Crowell77] Crowell R. H., Fox R. H.: "Introduction to knot theory", Springer New York Heidelberg, 1977, ISBN 0-387-90272-4, 3-54090272-4.

[Fung65] Fung Y. C.: "Foundations of solid mechanics", Prentice-Hall, 1965.

[Hasegawa92] Hasegawa T., Suehiro T., Takase T.: "A model-based manipulation system with skill-based execution". In: IEEE Trans. on Robotics and Automation, vol. 8, no. 5, pp. 535544, Oct., 1992.

[Higashijima98] Higashijima K., Onda H., Ogasawara T.: "Planning for wire obstacles avoidance using ultrasonic sensors". In: IEEE/RSJ International Conference on Intelligent Robots and Systems (IROS'98), Victoria, Oct., 1998.

[Hirai94] Hirai S., Wakamatsu H., Iwata K., "Modeling of deformable thin parts for their manipulation", In: Proc. Int. Conf. on Robotics and Automation (ICRA'94), vol. 4, pp. 2955-2960, San Diego, May, 1994.
[Inoue83] Inoue H., Inaba M.: "Hand-eye coordination in rope handling". In: Proc. of the First Int. Symp. on Robotics Research, pp. 163-174, New Hampshire, Sept., 1983.

[Kraus97] Kraus W., McCarragher B.J.: "Force fields in the manipulation of flexible materials", In: Proc. IEEE Int. Conf. on Robotics and Automation (ICRA'96), vol. 3, pp. 2352-2357, Minneapolis, April 1996.

[Morrow97] Morrow J. D., Khosla P. K.: "Manipulation task primitives for composing robot skills". In: Proc. of the IEEE Int. Conf. on Robotics and Automation (ICRA'97), Albuquerque, New Mexico, April 1997, pp. 33543359.

[Nakagaki95] Nakagaki H., Kitagaki K., Tsukune H.: "Study of insertion task of a flexible beam into a hole". In: Proc. of IEEE Int. Conf. on Robotics and Automation (ICCRA'95), vol. 1, pp. 330-3351, 1995.

[Nakagaki96] Nakagaki H., Kitagaki K., Ogasawara T., Tsukune H.: "Study of insertion task of a flexible beam into a hole by using visual tracking observed by stereo vision". In: Proc. of IEEE Int. Conf. on Robotics and Automation (ICRA'96), vol. 4, pp. 3209-3214, 1996.

[Nakashima95] Nakashima M., et al.: "Application of semi-automatic robot technology on hot-line maintenance work". In: IEEE Int. Conf. on Robotics and Automation, 1995, pp. 843-850.

[Onda95] Onda H., Hirukawa H., Takase K.: "Assembly motion teaching system using position/force simulator - Extracting a sequence of contact state transition". In: Proc. of the IEEE/RSJ International Conference on Intelligent Robots and Systems (IROS'95), vol. 1, pp. 9-16, 1995.

[Suehiro89] Suehiro T., Takase K.: "Representation and control of motion in contact and its application to assembly tasks". In: Proc. 5th Int. Symp. on Robotics Research, 1989.

[Wakamatsu95] Wakamatsu H., Hirai S., Iwata K., "Modeling of linear objects considering bend, twist, and extensional deformations". In: Proc. IEEE Int. Conf. on Robotics and Automation (ICRA'95), vol. 1, pp. 433-438, Nagoya, May, 1995.

[Wakamatsu97] Wakamatsu H., Matsumura T., Arai E., Hirai S.: "Dynamic Analysis of string objects deformation for their manipulation". In: Proc. IEEE/RSJ Int. Conf. on Intelligent Robots and Systems (IROS'97), pp. 196201, Grenoble, Sept., 1997.

[Zheng91] Zheng Y. F., Pei R., Chen C.: "Strategies for automation assembly of deformable objects". In: IEEE Int. Conf. on Robotics and Automation, 1991, pp. 2598-2603. 